\title{
SOLITON-LIKE LONGITUDINAL HOLES IN DEBUNCHED BEAMS PERPETUATED BY SPACE-CHARGE FORCES
}

\author{
S. Koscielniak, TRIUMF, 4004 Wesbrook Mall, Vancouver, B.C. Canada \\ S. Hancock \& M. Lindroos, CERN, CH-1211, Geneva 23, Switzerland
}

\begin{abstract}
Because the longitudinal space-charge forces within a local rarefaction of the particle density in phase space are focusing toward the centre of the perturbation, holes in beams might be expected to be self stabilized below transition energy. We shall report measurements[7, 8, 9] made at the CERN PS Booster (PSB) and computer simulations that demonstrate the surprising longevity of such perturbations on an unbunched charged-particle beam. The negative mass instability is not an explanation for this persistent beam structure and the usual wave theory for perturbations on unbunched beams (the Keil-Schnell criterion) cannot explain their stability. The holes are solutions of the non-linear Vlasov-Poisson equation for which we have found self-consistency and stationarity conditions; these have scaling-laws similar to the Keil-Schnell criterion.
\end{abstract}

\section{INTRODUCTION}

Localized longitudinal density perturbations on an unbunched charged-particle beam, have recently aroused interest. Colestock[1] and Spentzouris[2] have reported experimental observations and Schamel[4, 5, 6] has made theoretical studies and claims that the perturbations are soliton-like and cannot be predicted by the linearized Vlasov treatment used by Keil[3] and Schnell; we agree with this claim.

\subsection{CERN PS Booster Measurements}

The PS Booster is a proton synchrotron cycling between $50 \mathrm{MeV}$ and $1.4 \mathrm{GeV}$ kinetic energy. The machine operates with a harmonic $h=1$ fundamental rf system and in addition there is an $h=2 \mathrm{rf}$ system and a high harmonic cavity capable of generating harmonics from $h=12$ to $h=26$ at the injection energy. The Booster may also be operated in storage ring mode at $50 \mathrm{MeV}$. Beam is injected over a few turns (from 0.1 to 12 ) from a $200 \mathrm{MHz}$ linac.

A wide-band beam pick-up and a fast digital oscilloscope with deep memory are available for recording bunch shapes turn-by-turn. The data may be graphed as a waterfall display: a "bird's-eye" view of the conventional mountain-range display. A grey scale, from black to white, denotes relative particle density from most to least dense. The instrument and software is called the "tomoscope".

Linac bubbles The tomoscope was used to observe the debunched beam after injection. Beam structure which we refer to as "bubbles", may survive for at least $50 \mathrm{~ms}$. This longevity cannot be ascribed to narrow momentum width: no appreciable debunching of the bubbles implies an energy spread less than $5 \mathrm{eV}$, which is so small that the bubbles would be undetectable. The bubbles become more prominent at higher intensity (figure 1).

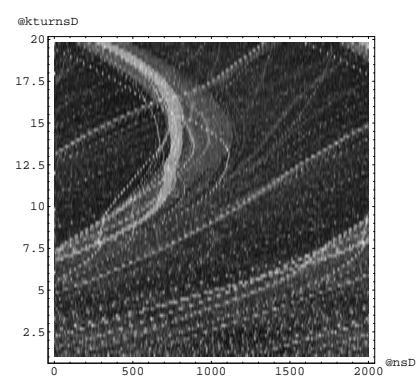

Figure 1: $3.6 \times 10^{12}$ protons, 1 -trace each 200 -turns, vertical span $31.7 \mathrm{~ms}$.

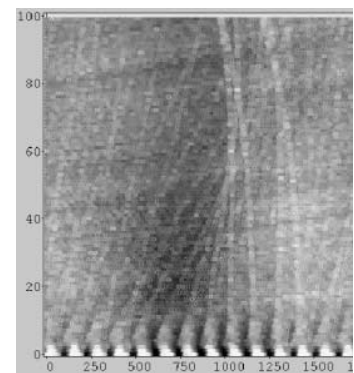

Figure 2: $2.4 \times 10^{12}$ protons, 1-trace/20-turns, span $3.3 \mathrm{~ms}$; abscissa ns.

\subsection{Periodic high-harmonic holes}

With the fundamental rf turned off, the high harmonic cavity can be used to introduce periodic holes with known properties. For example, $h=13$ holes having an energy spread of some $190 \mathrm{keV}$ were introduced into a beam of $3 \times$ $10^{12}$ protons. With the $h=13$ cavity switched off once the empty buckets were introduced, the top and bottom of the resulting holes should shear by a machine circumference each milli-second. No such shearing was detected.

\subsection{Momentum steering of holes}

Because the longitudinal space-charge force is proportional to the derivative of the line-current density, there is no force between holes until they contact. However, if there are differences in their central momenta, then holes will move relative to one another. To test this idea, one impresses a $h=1$ coherent energy modulation on the holes (using small voltage of short duration). Figure 2 shows a waterfall display after $\pm 60 \mathrm{keV}$ modulation at $h=1$; the holes' tracks converge in response to the modulation; and though some holes collide none coalesce.

\subsection{Momentum spread versus beam current}

A simple theoretical model (section 1.5) predicts a definite relation between the momentum spread of the holes and the beam current, because of a fine balance between shearing and focusing. The Booster experiments were 
adapted to test the theory using $h=18$ holes. In figure 3 , the tendency for holes to shear due to their momentum spread is well balanced against the focusing provided by space-charge forces and the tracks stay sharply defined. In figure 4 , the momentum spread of the holes has been enlarged and this enhances the shearing effect. Comparison of figures 4,5 and 6 , where the beam current is progressively increased, shows that space-charge focusing on holes can overcome the shearing due to momentum spread, leading to narrower and more self-sustained holes.

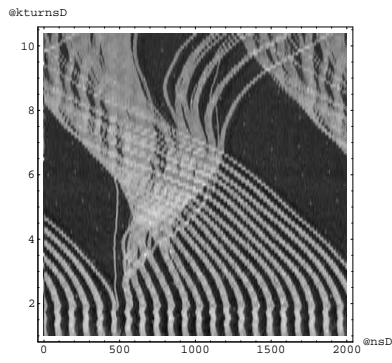

Figure 3: $1.8 \times 10^{12}$ protons, 1-trace each 100-turns, span $16.7 \mathrm{~ms}, V_{\mathrm{rf}}=0.3 \mathrm{kV}$.

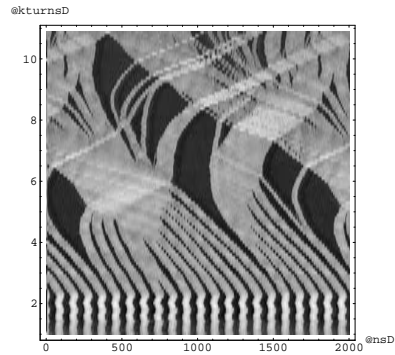

Figure 5: $2.4 \times 10^{12}$ protons, 1-trace each 100-turns, span $16.7 \mathrm{~ms}, V_{\mathrm{rf}}=0.55 \mathrm{kV}$.

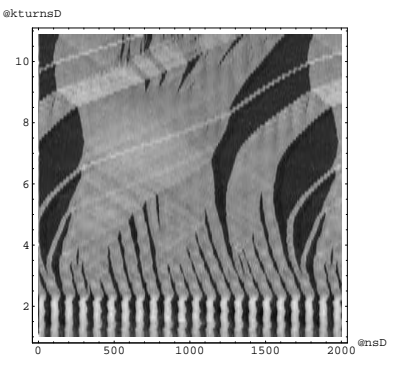

Figure 4: $1.8 \times 10^{12}$ protons, 1-trace each 100-turns, span $16.7 \mathrm{~ms}, V_{\mathrm{rf}}=0.6 \mathrm{kV}$.

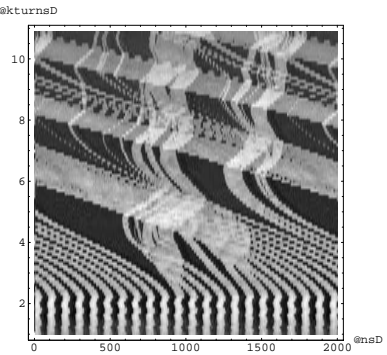

Figure 6: $3.2 \times 10^{12}$ protons, 1-trace each 100-turns, span $16.7 \mathrm{~ms}, V_{\mathrm{rf}}=0.6 \mathrm{kV}$.

\subsection{Simple theoretical model}

Suppose that space charge is the dominant longitudinal source of impedance. Let $g_{0}$ be the geometrical coupling parameter which depends on the ratio of beam and vacuum pipe transverse radii. Let $p$ and $q$ be canonical momentum and position. The Hamiltonian is of the form $H=p^{2} / 2+$ $Z \lambda(q)$ where $Z>0$ is the space-charge coupling constant and $\lambda$ is the line density. Self-consistency of a rectangular void of momentum width $2 \hat{p}$ and length $2 b$ radians, leads to the equilibrium condition:

$$
\left(\frac{\hat{p}}{P_{s}}\right)^{2}=\frac{g_{0}}{2 \pi \epsilon_{0}}\left(\frac{e}{\beta_{s}}\right)^{2} \frac{1}{E_{s}} \frac{N}{R_{s}} \frac{1}{\left[\left(\gamma_{s} / \gamma_{t}\right)^{2}-1\right]} \times \frac{f_{\text {empty }}}{f_{\text {filled }}} \frac{1}{2 b} .
$$

Here $E_{s}, P_{s}$ are energy and momentum, $\beta_{s} c=$ speed, $R_{s}=$ ring radius, $N=$ number of particles, $f_{\text {empty }}$ and $f_{\text {filled }}$ are the fractions of empty and filled phase space.

\section{COMPUTER SIMULATIONS}

Computer simulations were made using the LONG1D tracking programme[10]. Two types of system were stud- ied: (i) a periodic array of holes as the result of depositing $h=15$ empty rf buckets; and (ii) the behaviour of one or two rectangular holes. Example GIF animations may be found on the world-wide-web[11].

\subsection{Periodic holes}

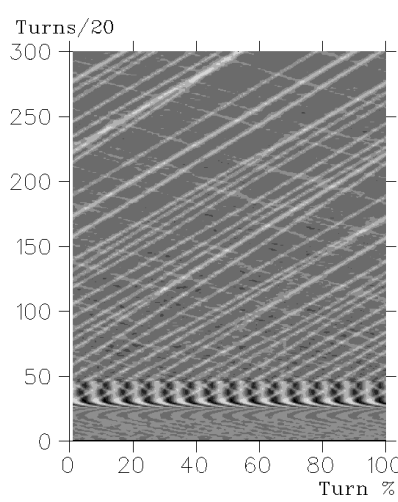

Simulations were made in which the beam was increased from zero to $4 \times 10^{12}$ protons. The zero intensity case shows the holes to debunch in $\leq 0.5 \mathrm{~ms}$. For $2 \times 10^{12}$ protons, the holes leave obvious tracks, though space-charge is not strong enough to prevent the holes from widening. For $4 \times 10^{12}$ protons (figure 7) the higher space-charge forces provide

Figure 7: $4 \times 10^{12}$ protons, $10^{6}$ macro-particles.

better focusing and the holes do not widen.

\subsection{Single rectangular holes}

Simulations were made for the cases of beam current one half and double the value required for stationarity. In the former, the hole debunches because the shearing due to momentum spread overcomes the space-charge focusing. In the latter (figure 8) the hole quickly breaks up into two smaller holes which thereafter appear stationary; the momentum spread is unaltered, but the charge in each is halved and so the stationarity condition is restored.

\subsection{Collision of two holes}

The case of two initially matched holes prepared with slightly different central momentum and initially separated in azimuth is shown in figure 9. The holes encounter and slide past one another. They do not coalesce.

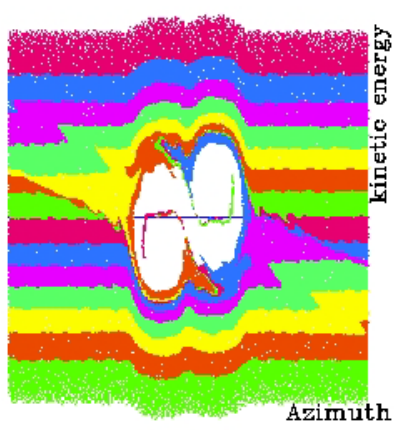

Figure 8: $10^{13}$ protons, $2.8 \times 10^{5}$ macro-particles.

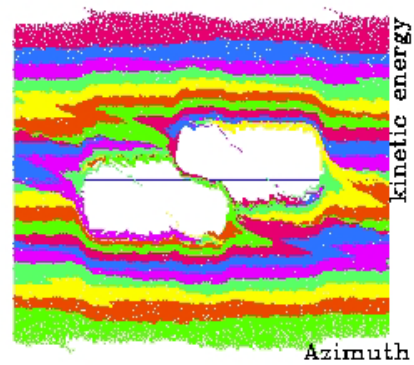

Figure 9: $5 \times 10^{12}$ protons, $2.2 \times 10^{5}$ macro-particles.

\section{VLASOV EQUATION}

We now examine the question of whether stationarity conditions such as (1) can be obtained from solution of the time-independent Vlasov equation. Let $\psi_{0}$ be a steady state density function and $\psi_{1}$ some perturbation. 


\subsection{Keil-Schnell type condition}

Let $Z$ represent the space-charge coupling to $\partial \lambda / \partial q$. The Vlasov equation is linearized and the time derivative set to zero, leading to

$$
\dot{p}\left(\psi_{1}\right) \frac{\partial \psi_{0}}{\partial p}+p \frac{\partial \psi_{1}}{\partial q}=0, \quad \frac{d p}{d t}=-Z \frac{\partial}{\partial q} \int_{-\infty}^{+\infty} \psi_{1}(p, q) d p .
$$

Hence the Keil-Schnell-type dispersion relation (for zero modulation frequency) is:

$$
Z \int_{-\infty}^{+\infty} \frac{1}{p} \frac{\partial \psi_{0}}{\partial p} d p=+1
$$

The PV integral is negative/positive if $\psi_{0}$ is locally a maximum/minimum. Thus for capacitive impedance $(Z>0)$, an inverted bell $\psi_{0}$ is required to perpetuate a perturbation below transition energy. Despite this prediction, the observed stable holes in the PSB debunched beam occur for a quasi-parabolic distribution with $\int\left(\partial \psi_{0} / \partial p\right) d p / p<0$.

\subsection{Whether or not to linearize}

When linearizing the Vlasov equation, it is customary to state that the perturbation $\psi_{1}$ is small compared with the steady state function $\psi_{0}$ and that the product $\dot{p} \psi_{1}$ can be neglected. However, the Vlasov equation is not concerned with the absolute amplitude of functions, but rather it is obsessed throughout with derivatives. Thus it is not the relative size of $\psi_{0}$ and $\psi_{1}$ that matters, but rather the relative size of their derivatives. For the case of a very localized perturbation the derivatives of $\psi_{1}$ may be overwhelmingly large compared with those of $\psi_{0}$. In this case, the perturbation decouples (almost) completely from the steady-state.

\subsection{Dispersion relation}

The stationary $\psi_{1}$ obeys equation (2) but with $\psi_{1}$ substituted in place of $\psi_{0}$. The dispersion relation is analogous to (3) but with $\psi_{1}$ substituted in place of $\psi_{0}$. Evidently, $\psi_{1}$ must be an inverted bell-shape, that is a hole, for the dispersion relation to be fulfilled (assuming $Z>0$ and below transition). The number of particles involved is $n=\int \psi_{1} d p d q$. The dispersion relation differs in two respects from Keil-Schnell: whereas the latter contains the full beam current $(\propto N)$ and the full momentum spread of the beam, the former contains only the current in the hole (proportional to $n$ ) and the momentum width of the hole.

\section{$3.4 \psi_{1}=P(p, t) Q(q, t)$}

We consider a more general impedance $R+i Z$ with resistive and reactive parts and $i=\sqrt{-1}$. Let $\sigma$ be a range parameter. We substitute the trial solution

$$
\psi_{1}=\frac{e^{-(p-\gamma t)^{2} / 2 \sigma^{2}}}{\sigma \sqrt{2 \pi}} \lambda_{1}\left(q-\gamma t^{2} / 2\right),
$$

into the time-dependent Vlasov equation. One finds the average momentum loss rate $\gamma=-R \int \lambda_{1}^{2}(q) d q / \int \lambda_{1}(q) d q$, and the residual

$$
Z \frac{\partial \lambda_{1}}{\partial q} \frac{1}{\sigma^{2}} \lambda_{1}(q)+\frac{\partial \lambda_{1}}{\partial q}=0
$$

Hence $\lambda$ must be the inverted "top hat" function within some range of $|q| \leq \Delta q$ and zero outside that range. $\lambda_{1}(q)$ is a negative constant: $\lambda_{1}=-\sigma^{2} / Z$. This condition implies a very strict relation between the momentum width $\sigma$ of a hole and its depth $\lambda_{1}$, such that progressively narrower holes $(\sigma<1)$ become very shallow and hard to detect.

Despite the decoupling of $\psi_{0}$ and $\psi_{1}$, the steady state distribution does impose some constraints on the perturbation. Because $\psi_{0} \geq 0$, the phase-space density must be high enough to support the perturbation. Thus for a hole of given momentum width, there is a critical density below which $\psi_{0}$ cannot act as a transport medium for solitons. The density $\psi_{1}$ is of order $n /(4 \sigma \Delta q)=-\sigma /(4 Z)$. The density $\psi_{0}$ is of order $N /(4 \pi \Delta p)$ where $\Delta p$ is the r.m.s. width of $\psi_{0}$. Hence the critical density is $N /(\pi \Delta p) \geq \sigma / Z$.

\section{CONCLUSION}

There is much experimental evidence at the CPS Booster of near-stationary longitudinal voids introduced accidentally by the linac or deliberately by high-harmonic rf. There is strong supporting evidence from computer simulations and theoretical analysis that the holes are perpetuated by space-charge forces. We have shown that the usual KeilSchnell criterion does not explain the voids, but rather suggested they decouple from the background distribution. We have derived conditions for stationarity of holes that satisfy the requirement of self-consistency - with the caveat that the phase-space density be high enough to support the solitons. However, we have not determined stability. A more complete discussion of these topics is given in Ref.[12]

\section{REFERENCES}

[1] P. Colestock \& L. Spentzouris: The Tamura Symposium, Austin, Texas, 1994 AIP Conf. Proc. 356 (AIP N.Y. 1996)

[2] Spentzouris, Ostiguy, Colestock: Direct measurement of diffusion rates..., Phys. Rev. Lett. Vol.76 pp. 620 (1996).

[3] E. Keil \& W. Schnell: CERN Report ISR-TH-RF/69-48.

[4] H. Schamel: Electron Holes, Ion Holes and Double Layers, Physics Reports, Vol.140 No.3, 1986.

[5] H. Schamel: Theory of Solitary Holes in Coasting Beams, Phys. Rev. Lett. Vol. 79 No. 15 pp. 2811-2814 (1997).

[6] H. Schamel: Kinetic theory of solitary waves... Proc. 7th European Particle Accelerator Conf., Vienna, pg. 1558 (2000).

[7] A. Blas et al: CERN PS/RF/Note 2000-016 (MD)

[8] A. Blas et al: CERN PS/RF/Note 2000-015 (MD).

[9] A. Blas et al: CERN PS/RF/Note 2000-014 (MD).

[10] S. Koscielniak: Longld Guide, TRIUMF TRI-DN-97-12.

[11] 9th ICFA Mini-Workshop, CERN, CH, March 2000; http://psdata.web.cern.ch/psdata/www/icfa9/ICFAWelcome

[12] S. Koscielniak et al: Longitudinal holes in debunched particle beams.., Phys.Rev. ST Accel. Beams, Vol.4, 044201. 